\title{
LARYNGOSCOPE INDUCED THERMAL INJURIES IN NEONATES: SOMETHING TO WATCH OUT FOR
}

\author{
R. Lall, R. Chakupurakal, A. Manzoor, T. Thangavelu, M. Ahmed \\ Paediatrics, Queen's Hospital Burton, Burton-upon-Trent, UK
}

Background and aims: During intubation injuries may occur to the patient either due to wrong intubation technique or faulty equipment. Injuries due to faulty equipment are less frequently documented than faulty technique. Thermal injuries induced by the heat of laryngoscope bulbs are very rare phenomena, only sporadically mentioned in the literature for adults. In neonates, so far only one case was described with oropharyngeal burn after intubation.

Methods: We did a biomedical analysis of the available laryngoscope bulbs to determine the risk of heat induced injuries during intubations. Our local medical engineering department carried out temperature measurements on all three presently available laryngoscopes on our neonatal unit. 3 types of blades/bulbs were analysed-non disposable fibre optic, disposable conventional and LED bulb laryngoscopes Measurements were performed under standardised conditions measuring first the baseline temperature (T0) at the tip of the light source before switching it on and after activation in the time intervals of 10 seconds (T10), 30 seconds, (T30), 1 minute (T60), 2 minutes (T120) and 3 minutes (T180).

Results: The mean temperature rise in the group of disposable conventional bulb laryngoscopes was significantly higher than in the fibre optic/disposable LED laryngoscopes.

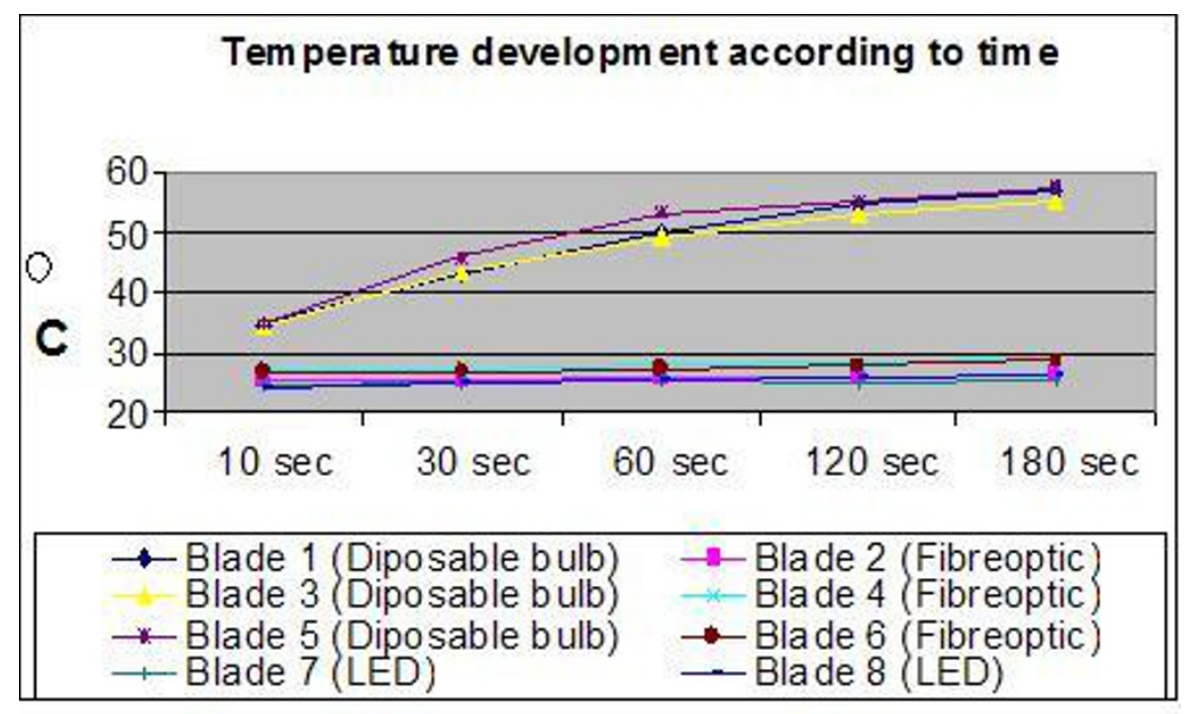

[laryngoscope temperature rise]

Conclusions: We therefore recommend using disposable LED blades which have significantly lower temperature rise as compared to conventional bulbs thereby decreasing the risk of thermal burns during intubations. 